\title{
Quantitative sensory testing measures individual pain responses in emergency department patients
}

This article was published in the following Dove Press journal:

Journal of Pain Research

24 May 2017

Number of times this article has been viewed

\section{Kevin J Duffy \\ Katharyn L Flickinger \\ Jeffrey $T$ Kristan \\ Melissa J Repine \\ Alexandro Gianforcaro \\ Rebecca B Hasley \\ Saad Feroz \\ Jessica M Rupp \\ Jumana Al-Baghli \\ Maria L Pacella \\ Brian P Suffoletto \\ Clifton W Callaway}

Department of Emergency Medicine, School of Medicine, University of Pittsburgh, Pittsburgh, PA, USA
Correspondence: Clifton W Callaway Department of Emergency Medicine, School of Medicine, University of Pittsburgh, 400A Iroquois, 3600 Forbes Avenue, Pittsburgh, PA 15260, USA

$\mathrm{Tel}+\mathrm{I} 4126479047$

Fax + I 4126476999

Email callawaycw@upmc.edu
Background: Refining and individualizing treatment of acute pain in the emergency department (ED) is a high priority, given that painful complaints are the most common reasons for ED visits. Few tools exist to objectively measure pain perception in the ED setting. We speculated that variation in perception of fixed painful stimuli would explain individual variation in reported pain and response to treatment among ED patients.

Materials and methods: In three studies, we 1) describe performance characteristics of brief quantitative sensory testing (QST) in 50 healthy volunteers, 2) test effects of $10 \mathrm{mg}$ oxycodone versus placebo on QST measures in 18 healthy volunteers, and 3) measure interindividual differences in nociception and treatment responses in 198 ED patients with a painful complaint during ED treatment. QST measures adapted for use in the ED included pressure sensation threshold, pressure pain threshold (PPT), pressure pain response (PPR), and cold pain tolerance (CPT) tests.

Results: First, all QST measures had high inter-rater reliability and test-retest reproducibility. Second, $10 \mathrm{mg}$ oxycodone reduced PPR, increased PPT, and prolonged CPT. Third, baseline PPT and PPR revealed hyperalgesia in 31 (16\%) ED subjects relative to healthy volunteers. In 173 (88\%) ED subjects who completed repeat testing 30 minutes after pain treatment, PPT increased and PPR decreased (Cohen's $\left.d_{z} 0.10-0.19\right)$. Verbal pain scores (0-10) for the ED complaint decreased by 2.2 (95\% confidence intervals [CI]: 1.9, 2.6) (Cohen's $\mathrm{d}_{\mathrm{z}}$ 0.97) but did not covary with the changes in PPT and PPR $(r=0.05-0.13)$. Treatment effects were greatest in ED subjects with a history of treatment for anxiety or depression (Cohen's $d_{z}$ 0.26-0.43) or with baseline hyperalgesia (Cohen's $\mathrm{d}_{\mathrm{z}} 0.40-0.88$ ).

Conclusion: QST reveals individual differences in perception of fixed painful stimuli in ED patients, including hyperalgesia. Subgroups of ED patients with hyperalgesia and psychiatric history report larger treatment effects on ED pain and QST measures.

Keywords: emergency department, quantitative sensory testing, hyperalgesia, opioid, anxiety, depression

\section{Introduction}

Refining treatment of acute pain in the emergency department (ED) is a high priority, given that painful complaints are the most common reasons for ED visits. ${ }^{1}$ Analgesic drugs are one of the most common drug classes prescribed in the ED with opioids representing an increasing proportion of these drugs. ${ }^{1-3}$ This situation potentially contributes to opioid overuse. ${ }^{4,5}$ Competing efforts focus on reducing inappropriate ED opioid prescribing ${ }^{6,7}$ as well as on reducing ED barriers and inequities in the effective treatment of pain. ${ }^{8-10}$ Despite this attention to ED pain treatment, few studies examine individualized pain treatment for emergency patients. ${ }^{11}$ 
Individualized treatment requires methods to reliably compare individual perceptions of painful stimuli and individual responses to analgesia in the ED. ${ }^{12}$ Measurement of pain and response to pain treatment is difficult because of the chaotic ED setting. In addition, modifiers of pain response include prior drug use, patient psychological traits and states, acute anxiety, and other stressors, which are common in the ED population. ${ }^{13-15}$ ED pain measurement relies primarily on verbal or visual numerical rating scales of pain intensity that are reliable for measuring within-subject effects. ${ }^{16-19}$ However, these pain scales do not permit interindividual comparisons, and single numerical scales may not capture the clinically important discomfort or tolerability of pain of a given intensity. ${ }^{20}$

To move toward more personalized treatment of acute pain in the ED, we adapted several quantitative sensory testing (QST) measures from the literature into a battery for comparing individual sensory thresholds and responses to fixed painful stimuli in ED patients. We selected QST measures with the long-term goal of using these tools in multiple busy EDs. Therefore, we selected measures that were simple, portable, and with sufficiently brief administration to allow repeated assessments. We prioritized tests that did not require specialized or expensive equipment unlikely to be found in an ED.

This project consisted of two phases: 1) describing the performance characteristics of the QST battery in healthy volunteers and 2) describing the feasibility of the QST regimen in a cohort of ED patients with painful complaints. We hypothesized that ED patients have between individual variations in perception of fixed painful stimuli prior to treatment, and that ED treatment alters perception of fixed painful stimuli. Because we were interested in the global nociceptive responses of each subject, we tested perception to fixed stimuli in a single anatomical location, remote from the site of pain from the ED complaint. As secondary aims, we explored whether there were associations between subjective pain relief and baseline QST measures, changes in QST with treatment, or clinical features of patients.

\section{Materials and methods}

The University of Pittsburgh Institutional Review Board reviewed and approved the healthy volunteer studies and the ED studies separately. All subjects provided written informed consent prior to participation. For laboratory studies, we provided informed consent documents to interested participants in advance of any testing, and the potential participant could review the document for as long as they wished. In the ED studies, a member of the treating clinical team asked potential participants if they would be interested in participating in research. If they agreed, a researcher verbally described the study at bedside including demonstration of pressure algometry on the researcher's own hand. The potential subject reviewed the consent form simultaneously with opportunity to ask questions. No data were collected until after the potential subject signed the consent form. We did not permit the research study to delay ED treatment. Therefore, if a potential subject wished for a longer time to consider participation, they were not included in the study.

\section{Participants}

For healthy volunteer studies, we recruited male and female adults ( $\geq 18$ years of age) from the University of Pittsburgh community using a printed advertisement and word of mouth. We excluded subjects with a history of neuropathy, scars on their right hand, or current use of pain-relieving drugs. For the study of oxycodone, we also excluded subjects with prior intolerance of opioids or pregnancy. The initial healthy volunteer cohort was not compensated for participation, but subjects in the oxycodone study received $\$ 20$ for participation.

For ED studies, we recruited a convenience sample of adult ( $\geq 18$ years of age) patients from the EDs of three teaching hospitals in Pittsburgh with $\sim 70,000,54,000$, and 23,000 patient visits per year. The two busiest EDs are Level 1 Trauma Centers. A researcher approached alert patients who were being evaluated for a painful condition (initial pain score $\geq 4$ using a verbal scale from 0 to 10 with anchors of $0=$ "no pain" and $10=$ "worst possible pain") and for whom the treating team planned to administer an analgesic. The researchers did not alter the treating team analgesic plan in any way. We excluded subjects if they had diagnosed neuropathy or had scars or injury on their right hand. ED subjects received no compensation.

\section{QST measurements}

The primary investigators trained all research specialists who conducted QST. The specific measurements for this study included pressure-sensation threshold (PST), pressure-pain threshold (PPT), pressure-pain response (PPR), and coldpain threshold (CPT). Each specialist practiced administration of the QST measures on the investigators and on each other until proficient. Investigators observed testing by the specialists periodically during the study to assure that there was no drift in practice.

\section{Pressure-sensation threshold}

PST is the smallest perceptible pressure from Von Frey fibers $(0.02-40 \mathrm{~g})$. With the subject's eyes closed or turned away, we pressed fibers to the skin over the soft tissue between the 
distal ends of the metacarpals of the index and long fingers of the right hand. Subjects were told, "Tell me when you feel me touching you". If the subject reported touch, we then tested the next smaller Von Frey fiber, whereas if the subject did not report touch, we tested the next larger Von Frey fiber. This process continued until we identified the smallest detectable Von Frey fiber for that subject. Lower scores indicate increased ability to perceive the touch stimulus.

\section{Pressure-pain threshold}

We measured PPT by applying a pressure algometer with a $1 \mathrm{~cm}^{2}$ round rubber foot (Wagner Instruments, Greenwich, CT, USA) in the webspace between distal metacarpal of the index finger and the long finger of the right hand. The pressure was increased at a rate of $10 \mathrm{~N} /$ second. We instructed subjects to indicate, "when the pressure stimulus becomes painful or uncomfortable". PPT was the pressure reading at that moment, with lower scores indicating lower threshold for perceiving pain. We explained to subjects to report the time when the subject wished the stimulus to stop, regardless of whether they could tolerate the stimulus for a longer time. In pilot testing, when we used the shorter phrase, "when the pressure stimulus becomes painful", subjects sometimes attempted to see how long they could endure the stimulus after it became noxious.

\section{Pressure-pain response}

PPR is reported pain of two pressures applied for 4-5 seconds using the pressure algometer between distal metacarpal of the index finger and the long finger of the right hand. We tested pressures of 50 and $75 \mathrm{~N} / \mathrm{cm}^{2}$. We asked subjects, "How uncomfortable is this pressure?" using a verbal scale from 0 to 10 with anchors of $0=$ "no pain" and $10=$ "worst possible pain". Subjects then marked their pain on a $10-\mathrm{cm}$ long Visual Analog Scale (VAS) with anchors of "no pain" and "worst possible pain." If the subject could not tolerate increasing the pressure to the intended level, we recorded verbal 10 and VAS $10 \mathrm{~cm}$. Verbal scores were always reported before VAS. Higher scores indicate increased intensity of perception of the stimulus.

\section{Cold-pain threshold}

CPT is the duration of cold exposure after which immersion of the right hand in cold water becomes uncomfortable. Each subject placed the right hand into a mixture of crushed ice and water in a plastic cylinder (14 cm diameter $\times 22 \mathrm{~cm}$ deep). We instructed subjects to keep their hand in the center of the cylinder, away from the edges. We did not agitate or mix the slurry during testing. In bench-top experiments, the water temperature in the center of the container was $0.5-1.7^{\circ} \mathrm{C}$ (mean $0.7^{\circ} \mathrm{C}, \mathrm{SD} 0.4^{\circ} \mathrm{C}$ ), and the water temperature at the edge of the container was $1.7-4.6^{\circ} \mathrm{C}$ (mean $2.8^{\circ} \mathrm{C}$, SD $1.1^{\circ} \mathrm{C}$ ). We instructed subjects to report, "when the cold stimulus becomes painful or uncomfortable." CPT was recorded as the time in seconds from immersion until the subject reported pain, with shorter durations indicating a lower threshold for response. Explanations of "painful or uncomfortable" were identical to those for PPT.

\section{Clinical information}

ED subjects completed a baseline survey that included current and prior history of treatment for anxiety and depression, drug use, and analgesic use. History of treatment for anxiety and depression was a dichotomous variable taken from the medical record problem list: we did not attempt to elicit detailed histories. Subjects also answered the Patient Health Questionnaire-2 (PHQ-2) ${ }^{21}$ and Generalized Anxiety Disorder scale-2 (GAD-2) 22 screening questions about state depressive symptoms and anxiety symptoms, respectively, with scores $\geq 3$ considered positive. Analgesic treatment was determined from the medical record. Investigators asked subjects to indicate the location of pain. After the encounter, we categorized the ED painful complaint as musculoskeletal (e.g., back pain, fracture, sprain), visceral (e.g., abdominal pain, urinary pain), soft tissue (e.g., abscess, cellulitis), headache, or diffuse pain.

\section{Study design}

We conducted three separate studies in separate subjects.

\section{Study I: QST performance characteristics}

The first study characterized the performance characteristics of PST, PPT, PPR, and CPT in 50 healthy volunteers. Each measure was recorded for each participant twice on the same day. Between testing, to account for changes in skin temperature with the CPT testing, we asked each subject to report when his or her hand was "back to normal" before repeat testing. This allowed about 10 minutes between testing sessions. Each test was repeated in the same order. In a subset of subjects, different investigators conducted the two tests to determine the inter-rater reliability.

\section{Study 2: oxycodone effects on QST measures}

The second study determined the effects of analgesic drugs on PST, PPT, PPR, and CPT in healthy volunteers. To achieve this aim, we tested the hypotheses that oxycodone would increase PST, PPT, and CPT, and decrease PPR. Each subject participated in two separate testing days at least 2 days apart. 
We asked subjects to fast for 6 hours prior to testing. On each day, subjects ingested identical appearing capsules that were either placebo or $10 \mathrm{mg}$ oxycodone, with each subject participating in both conditions. A pharmacist randomly assigned order of drugs using a computer, and both subjects and investigator were blinded to drug order. The pharmacy revealed drug identity after all data from all subjects were collected. Investigators recorded each sensory measure at baseline (two separate measurements) and at 30, 60, and 90 minutes after ingestion of a test drug.

\section{Study 3: QST in ED patients}

The third study determined the changes of PPT and PPR in subjects during treatment of painful conditions in the ED. Based on the length of time required to collect PST and CPT in the healthy volunteer studies, we deemed these measures to be impractical for the initial study in the ED. A researcher recorded PPT and PPR after a nurse and physician had evaluated the subject and an analgesic had been ordered but not yet administered. All subjects were aware that treatment for their painful condition already had been ordered and was not contingent on QST testing. The researcher recorded PPT and PPR a second time 30-60 minutes after analgesic had been administered. We selected a 30-minute interval because ED patients frequently receive multiple interventions during longer intervals.

\section{Statistical analysis}

Doses of opioid medications were converted to intravenous morphine equivalent dose (MEQ) using conversions from the literature: $1 \mathrm{mg}$ hydromorphone: $4 \mathrm{mg}$ morphine; $5 \mathrm{mg}$ oxycodone:2 $\mathrm{mg}$ morphine; and $5 \mathrm{mg}$ hydrocodone: $2 \mathrm{mg}$ morphine. ${ }^{23,24}$

Continuous variables were reported as means and standard deviations (SD) if normally distributed or medians and interquartile ranges (IQR) if not normally distributed. Proportions were expressed as percentages.

\section{Study I: QST performance characteristics}

In healthy volunteers, we measured associations between test outcomes using pairwise Pearson's correlations. We determined test-retest reliability of each measure by calculating Pearson's correlations, intraclass correlations, and BlandAltman plots between first and second trials. Bland-Altman statistics included test bias and 95\% confidence intervals (95\% CI) of agreement. ${ }^{25}$ We determined inter-rater reliability of each measure by calculating these measures between trials for each subject conducted by two different investigators.

\section{Study 2: oxycodone effects on QST measures}

The oxycodone study repeated measurements within subjects. Therefore, we compared the change over time in measured variables using mixed-effects regression, treating subjects as a random effect. Contrasts focused on the interaction between time and drug, and we compared values in each group to the mean baseline value for the same day. Study design dictated measurement times, and time was treated as a fixed effect. We used Bonferroni corrections to account for multiple comparisons within and between variables.

\section{Study 3: QST in ED patients}

For the ED subjects, we compared the distributions of QST measures with the distributions in the healthy volunteer cohort using two-sample Kolmogorov-Smirnov tests. To compare QST measures before and after ED treatment, we examined pairwise $t$-tests on the log transform of PPT. Other variables were not normally distributed, even when transformed, and we used nonparametric Wilcoxon matched pairs signed rank test to evaluate for change. We used Bonferroni correction to account for testing multiple variables within each subject. We examined a subgroup of subjects with hypersensitivity or hyperalgesia, arbitrarily defined as scores on PPR that were outside the ranges observed in healthy volunteers.

To explore whether effect sizes differed between subgroups and across measures, we calculated mean differences (with 95\% CI) between scores before and after treatment and also Cohen's $d_{z}$, which is a normalized measure of effect size for comparing a repeated measurement within subjects. Suggested benchmarks exist for interpreting effects as small $(d=0.2)$, medium $(d=0.5)$, or large $(d=0.8) .{ }^{26}$ Because these analyses were exploratory, we did not calculate any inferential statistics to compare effects in subgroups. Uncorrected $t$-test or chi-square comparisons of demographic or clinical characteristics described differences in subgroups.

\section{Sample size}

Sample size of 50 for the initial cohort of healthy volunteers was sufficient to provide estimates of mean and variance for measures with $90 \%$ power to detect correlations, $r>0.4$, between measures. We chose sample size of 20 for the study of oxycodone in healthy volunteers based on the SD and test-retest correlation (0.88) for VAS scores for PPR observed in the healthy volunteer study to provide about $90 \%$ power (beta $=0.88$ ) to detect a $10-\mathrm{mm}$ change in pain scores. We aimed to recruit 200 subjects for the ED cohort to estimate the proportion of subjects who would complete testing with $5 \%$ CIs. We estimated that this sample size should provide $90 \%$ 
power to detect correlations, $r>0.2$, between QST measures, and subjective pain relief.

Alpha error rates of 0.05 were used for significance. Analyses were conducted in STATA (v 13.1, College Station, TX, USA).

\section{Results}

\section{Study I: QST performance characteristics in healthy volunteers}

Fifty subjects (18 females, 32 males, mean [SD] age: 31 [12] years) participated in QST measurements. All subjects completed two tests: 16 by separate testers and 34 by the same tester. Reproducibility of each test appears in Table 1. Test-retest correlation was excellent, $r>0.80$, for all tests with small bias. Inter-rater reliability was excellent $(r>0.80)$ with small bias, for all tests except for PST, for which correlation was moderate $(\mathrm{r}=0.52)$.

Pairwise correlations between individual tests appear in Table 2. Verbal report and VAS for pain were highly correlated for $50 \mathrm{~N} / \mathrm{cm}^{2}(\mathrm{r}=0.97)$ and $75 \mathrm{~N} / \mathrm{cm}^{2}(\mathrm{r}=0.98)$. All of the pressure tests (PPT: 50 and $75 \mathrm{~N} / \mathrm{cm}^{2}$ ) were associated with each other. However, the associations between pressure tests, CPT, and PST were weak.

\section{Study 2: oxycodone effects on QST measures in healthy volunteers}

From 20 subjects who consented to begin the study, 18 completed all aspects of the protocol ( 8 males and 10 females, mean [SD] age of 30 [10] years, mean [SD] body mass of $67.3[11.2] \mathrm{kg}$ ). The two nonparticipating subjects did not schedule their laboratory visit after randomization and contributed no data. The two visits for each subject were 2-71 days apart (median, IQR: 13, 7-28 days).

Baseline QST measures were similar to the initial cohort of healthy volunteers, and measures varied little between testing days. For PPR, the VAS and verbal score were highly correlated ( $\mathrm{r}=0.94-0.97)$, and only the VAS is presented.

Treatment with $10 \mathrm{mg}$ oxycodone compared to placebo reduced PPR at $50 \mathrm{~N} / \mathrm{cm}^{2}(p=0.0038)$ and at $75 \mathrm{~N} / \mathrm{cm}^{2}$ $(p<0.0001)$, increased PPT $(p<0.001)$, and prolonged CPT $(p=0.0022)$ (Figure 1). There was an interaction between drug and time for PPR at $50 \mathrm{~N} / \mathrm{cm}^{2}(p=0.0008)$, at $75 \mathrm{~N} / \mathrm{cm}^{2}$ $(p<0.0001)$, and CPT $(p=0.0002)$ : PPR decreased and CPT increased from baseline at 60 and 90 minutes after oxycodone.

\section{Study 3: QST in ED patients}

From 205 ED patients with acute painful complaints who consented to begin the study, 197 subjects agreed to participate with QST measures and 173 (88\%) completed measurements both prior to and after pain treatment. Reasons for not completing the study are depicted in Figure 2, and characteristics of the subjects who completed the study did not differ from the total sample (Table 3).

A history of treatment for anxiety and depression was common (46\%), with some subjects (25\%) receiving current

Table I Reliability and reproducibility of QST measures in healthy volunteers $(n=50)$

\begin{tabular}{|c|c|c|c|}
\hline QST Measure & $\begin{array}{l}\text { Correlation } \\
\text { coefficient }\end{array}$ & $\begin{array}{l}\text { Intraclass correlation } \\
(95 \% \mathrm{Cl})\end{array}$ & $\begin{array}{l}\text { Bland-Altman bias } \\
\text { (95\% Cl of agreement) }\end{array}$ \\
\hline \multicolumn{4}{|c|}{ Between-test comparison $(\mathrm{N}=\mathbf{5 0})$} \\
\hline PST & $0.81 * * *$ & $0.66(0.47,0.79)$ & $-0.02(-0.21,0.18) g$ \\
\hline PPT & $0.81 * * *$ & $0.78(0.64,0.87)$ & $-2(-2 I, \mid 7) \mathrm{N} / \mathrm{cm}^{2}$ \\
\hline PPR $50 \mathrm{~N} / \mathrm{cm}^{2}$ (Verbal) & $0.86 * * *$ & $0.84(0.74,0.91)$ & $0.4(-1.8,2.6)$ \\
\hline PPR $50 \mathrm{~N} / \mathrm{cm}^{2}$ (VAS) & $0.88 * * *$ & $0.87(0.78,0.92)$ & $0.4(-1.7,2.4) \mathrm{cm}$ \\
\hline PPR $75 \mathrm{~N} / \mathrm{cm}^{2}$ (Verbal) & $0.88 * * *$ & $0.88(0.80,0.93)$ & $0.1(-2.0,2.2)$ \\
\hline PPR $75 \mathrm{~N} / \mathrm{cm}^{2}$ (VAS) & $0.88 * * *$ & $0.88(0.80,0.93)$ & $0.04(-2.1,2.2) \mathrm{cm}$ \\
\hline CPT & $0.97 * * *$ & $0.93(0.89,0.96)$ & $-4(-73,65)$ seconds \\
\hline \multicolumn{4}{|c|}{ Between-rater comparison $(\mathrm{N}=16)$} \\
\hline PST & $0.52 *$ & $0.44(-0.03,0.76)$ & $-0.02(-0.18 .0 .14) \mathrm{g}$ \\
\hline PPT & $0.81 * *$ & $0.79(0.52,0.92)$ & $-3.1(-23.17) \mathrm{N} / \mathrm{cm}^{2}$ \\
\hline PPR $50 \mathrm{~N} / \mathrm{cm}^{2}$ (Verbal) & $0.93 * * *$ & $0.93(0.82,0.98)$ & $0.1(-1.6,1.7)$ \\
\hline PPR $50 \mathrm{~N} / \mathrm{cm}^{2}$ (VAS) & $0.96 * * *$ & $0.96(0.88,0.98)$ & $0.1(-I . I, I .4) \mathrm{cm}$ \\
\hline PPR $75 \mathrm{~N} / \mathrm{cm}^{2}$ (Verbal) & $0.93 * * *$ & $0.93(0.83,0.98)$ & $0.1(-1.7,1.8)$ \\
\hline PPR $75 \mathrm{~N} / \mathrm{cm}^{2}$ (VAS) & $0.92^{* * *}$ & $0.92(0.78,0.97)$ & $-0.3(-2.2,1.6) \mathrm{cm}$ \\
\hline CPT & $0.94 * * *$ & $0.83(0.59,0.94)$ & $-9(-112,95)$ seconds \\
\hline
\end{tabular}

Note: $*_{p}<0.05 ; * * p<0.01 ; * * * p<0.0001$

Abbreviations: $\mathrm{Cl}$, confidence intervals; CPT, cold pain tolerance; PPR, pressure pain response; PPT, pressure pain threshold; PST, pressure-sensation threshold; QST, quantitative sensory testing; VAS, Visual Analog Scale. 
Table 2 Between-measure correlation of QST measures in healthy volunteers $(n=50)$

\begin{tabular}{|c|c|c|c|c|c|c|}
\hline QST Measure & PST & PPT & $\begin{array}{l}\text { PPR } 50 \mathrm{~N} / \mathrm{cm}^{2} \\
\text { (Verbal) }\end{array}$ & $\begin{array}{l}\text { PPR } 50 \mathrm{~N} / \mathrm{cm}^{2} \\
\text { (VAS) }\end{array}$ & $\begin{array}{l}\text { PPR } 75 \mathrm{~N} / \mathrm{cm}^{2} \\
\text { (Verbal) }\end{array}$ & $\begin{array}{l}\text { PPR } 75 \mathrm{~N} / \mathrm{cm}^{2} \\
\text { (VAS) }\end{array}$ \\
\hline PPT & 0.03 & & & & & \\
\hline PPR $50 \mathrm{~N} / \mathrm{cm}^{2}$ (Verbal) & 0.20 & $-0.30 *$ & & & & \\
\hline PPR $50 \mathrm{~N} / \mathrm{cm}^{2}$ (VAS) & 0.21 & $-0.28^{*}$ & $0.97 * * *$ & & & \\
\hline PPR 75 N/cm² (Verbal) & 0.11 & $-0.39 * *$ & $0.83 * * *$ & $0.80 * * *$ & & \\
\hline PPR 75 N/cm² (VAS) & 0.16 & $-0.35^{*}$ & $0.83 * * *$ & $0.8 I^{* * *}$ & $0.98 * *$ & \\
\hline CPT & -0.13 & 0.20 & -0.02 & -0.05 & -0.03 & -0.01 \\
\hline
\end{tabular}

Note: $*_{p}<0.05 ; * * p<0.01 ; * * * p<0.0001$.

Abbreviations: CPT, cold pain tolerance; PPR, pressure pain response; PPT, pressure pain threshold; PST, pressure sensation threshold; QST, quantitative sensory testing; VAS, Visual Analog Scale.
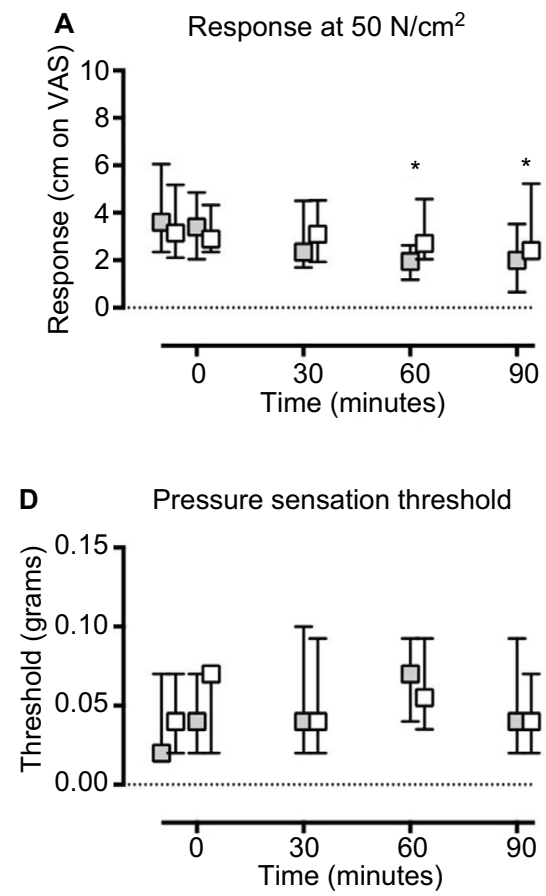
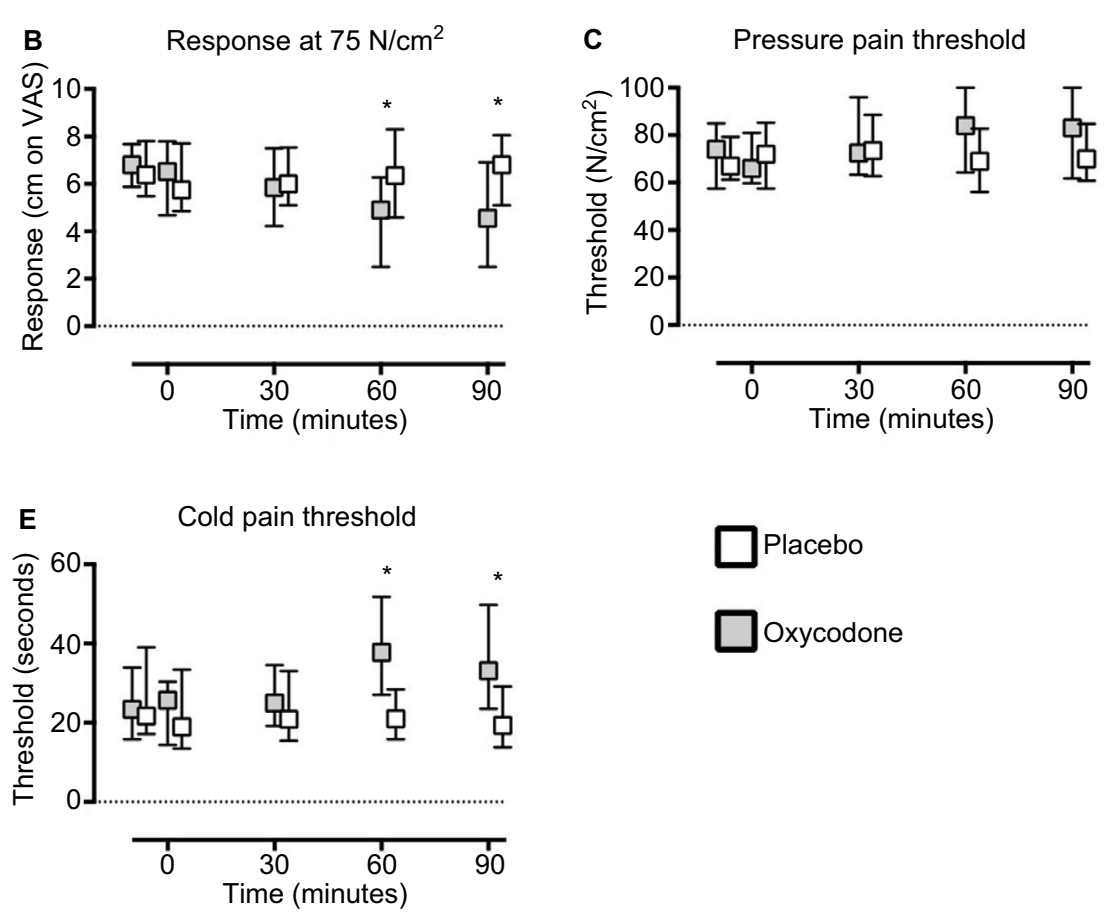

$\square$ Placebo $\square$ Oxycodone

Figure I Response of QST measures to $10 \mathrm{mg}$ oxycodone in healthy volunteers.

Notes: Group median and intraquartile ranges are depicted for pressure pain response at $50 \mathrm{~N} / \mathrm{cm}^{2}(\mathbf{A})$, at $75 \mathrm{~N} / \mathrm{cm}^{2}(\mathbf{B})$, pressure pain threshold $(\mathbf{C})$, pressure sensation threshold (D), and cold pain threshold (E). After oxycodone, the response at $50 \mathrm{~N} / \mathrm{cm}^{2}$, response at $75 \mathrm{~N} / \mathrm{cm}^{2}$, and cold pain response were decreased relative to baseline at 60 and 90 minutes $(* p<0.05)$.

Abbreviations: QST, quantitative sensory testing.

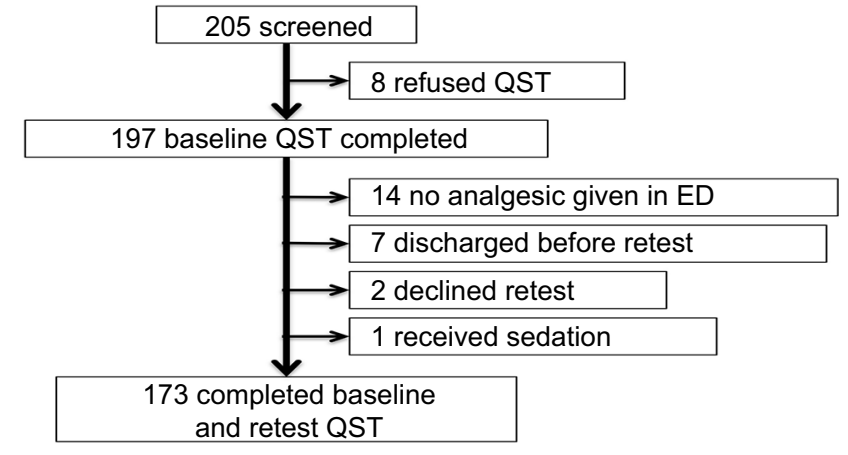

Figure 2 Subjects recruited from ED patients, and reasons for exclusion. Abbreviations: ED, emergency department; QST, quantitative sensory testing.

treatment for anxiety or depression. History or current treatment was associated with higher scores on the PHQ-2 and
GAD-2 (chi-square $=31-33, p<0.0001)$. Anxiety and depressive symptoms occurred together, with scores on PHQ-2 and GAD-2 positively correlated $(\mathrm{r}=0.67, p<0.0001)$. Of 173 subjects, 91 (53\%) scored $<3$ on both GAD-2 and PHQ-2 and $47(27 \%)$ scored $\geq 3$ on both. Musculoskeletal pain and visceral pain were most common, and pain was most often located in the extremities, abdomen, and back.

Baseline PPT and PPR measurements in ED subjects were numerically similar to the measurements in healthy volunteers, but the distribution of PPT $(p=0.004)$ and PPR at $75 \mathrm{~N} / \mathrm{cm}^{2}$ differed ( $p=0.006$ ) (Figure 3). Many subjects (34\%) would not allow the investigator to complete the $75 \mathrm{~N} / \mathrm{cm}^{2}$ PPR and a subset (9\%) would not tolerate the $50 \mathrm{~N} / \mathrm{cm}^{2} \mathrm{PPR}$, resulting in a score of $10 \mathrm{~cm}$ for those measures. Baseline 
Table 3 Characteristics of ED subjects

\begin{tabular}{|c|c|c|}
\hline Characteristics & Entire ED cohort & Subjects with repeated measures \\
\hline Number & 197 & 173 \\
\hline Mean age, years (SD) & $38(14)$ & $38(14)$ \\
\hline Male sex, $(\%)$ & 38 & 37 \\
\hline \multicolumn{3}{|l|}{ Race, \% } \\
\hline White & 54 & 57 \\
\hline African-American & 37 & 35 \\
\hline Asian & 1.5 & 1.7 \\
\hline Native American/Pacific Islander & 1.0 & 1.2 \\
\hline Biracial & 5 & 5 \\
\hline Declined to report & 1.5 & 0 \\
\hline Hispanic & 4.6 & 5.2 \\
\hline \multicolumn{3}{|l|}{ Education, \% } \\
\hline Less than high school & 10 & 9 \\
\hline High school/GED & 34 & 34 \\
\hline Some college & 30 & 31 \\
\hline College degree & 16 & 17 \\
\hline Postgraduate & 4 & 4 \\
\hline Technical/vocational school & 4 & 4 \\
\hline Declined to report & 3 & 2 \\
\hline \multicolumn{3}{|l|}{ Medical History } \\
\hline History of anxiety or depression, $\%$ & 46 & 46 \\
\hline Current treatment for anxiety or depression, $\%$ & 26 & 25 \\
\hline Current tobacco use, $\%$ & 53 & 55 \\
\hline Current alcohol use, \% & 52 & 53 \\
\hline Current marijuana use, $\%$ & 23 & 26 \\
\hline Current nonmedical opioid use, $\%$ & 0.5 & 0.6 \\
\hline Current medical opioid use, $\%$ & 12 & II \\
\hline Initial pain score, median (IQR) & $8(7,9)$ & $8(7,10)$ \\
\hline \multicolumn{3}{|l|}{ Character of pain, $\%$} \\
\hline Musculoskeletal & 50 & 47 \\
\hline Visceral & 38 & 40 \\
\hline Soft tissue & 8 & 8 \\
\hline Headache & 1.5 & 1.7 \\
\hline Diffuse & 1.5 & 1.7 \\
\hline \multicolumn{3}{|l|}{ Location of pain* } \\
\hline Extremities & 38 & 37 \\
\hline Abdominal & 35 & 38 \\
\hline Back & 33 & 35 \\
\hline Head & II & 12 \\
\hline Neck & 12 & 12 \\
\hline Chest & 7 & 6 \\
\hline
\end{tabular}

Notes: *Total $>100 \%$ because subjects could indicate multiple locations. The entire ED cohort provided some QST and baseline data. The subset of subjects with repeated measures also provided data after treatment with an analgesic.

Abbreviations: ED, emergency department; GED, General Educational Development; IQR, interquartile ranges; QST, quantitative sensory testing; SD, standard deviation.

PPR at $50 \mathrm{~N} / \mathrm{cm}^{2}$ was associated with the initial ED pain score $(\mathrm{r}=0.20, p=0.0052)$, but other QST measures were not associated with pain score.

As an exploratory analysis, we defined hyperalgesia to QST as a baseline PPR to 50 and $75 \mathrm{~N} / \mathrm{cm}^{2}$ of $9 \mathrm{~cm}$ or greater, because these values were outside of the range observed in healthy volunteers (Figure 3). Hyperalgesia occurred in 31 of 196 subjects (16\%) and in 23 of 173 (13\%) subjects with repeated measures. Mean (SD) PPT was lower (30 [8] vs. 48 $\left.[18] \mathrm{N} / \mathrm{cm}^{2}, p<0.0001\right)$ and mean (SD) initial pain score was higher in these subjects $(8.5[1.7]$ vs. $7.8[1.8], p=0.043)$. This subgroup of subjects was older (mean [SD] of 43 [12] vs. 37 [14] years, $p=0.05$ ), higher proportion of African-American ( $60 \%$ vs. $33 \%, p=0.033$ ), and more frequently with prior treatment ( $69 \%$ vs. $44 \%, p=0.014)$ or under current treatment ( $45 \%$ vs. $24 \%, p=0.022$ ) for anxiety or depression. Other measures did not differ for this subgroup.

\section{Response to pain treatment in emergency department}

Pain was treated with 2 or $4 \mathrm{mg}$ MEQ of opioid in $98(56 \%)$ cases (median dose 2, IQR 2-4). One subject received $0.8 \mathrm{mg}$ 
A

Response at $50 \mathrm{~N} / \mathrm{cm}^{2}$

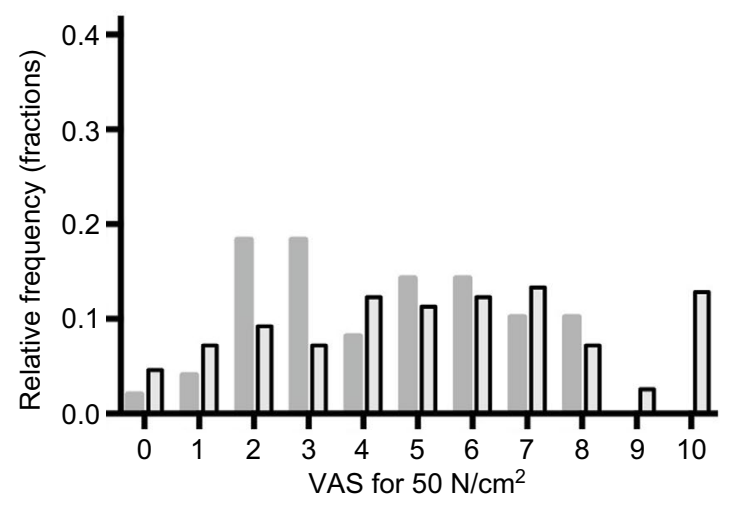

C

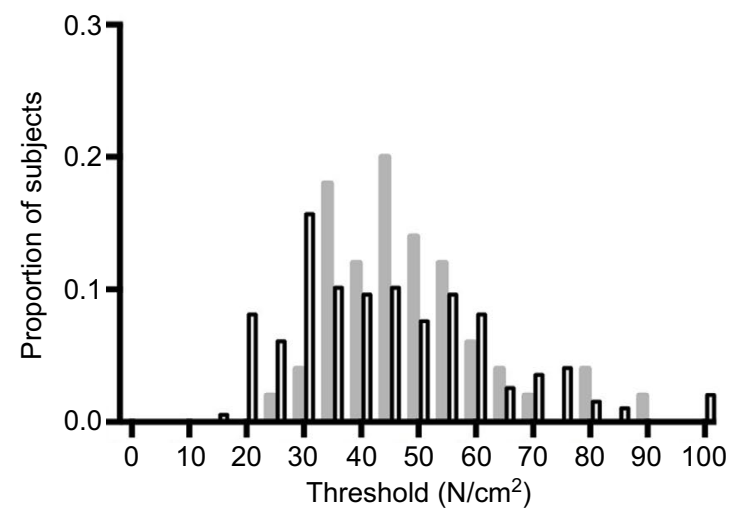

B

Response at $75 \mathrm{~N} / \mathrm{cm}^{2}$

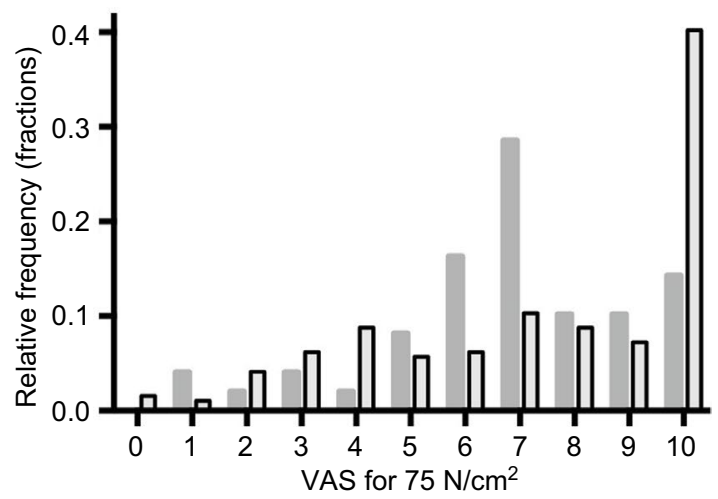

Healthy volunteer

$\square$ ED subject

Figure 3 Distribution of pain response at $50 \mathrm{~N} / \mathrm{cm}^{2}(\mathbf{A})$ and at $75 \mathrm{~N} / \mathrm{cm}^{2}(\mathbf{B})$ and pressure pain threshold (C) in healthy volunteers and in ED subjects (VAS in cm). Notes: The distribution of pressure pain thresholds and pain response at $75 \mathrm{~N} / \mathrm{cm}^{2}$ differed between cohorts. Lower pain thresholds were more frequent among ED subjects, and maximal pain responses ( $>9 \mathrm{~cm}$ on VAS) were more frequent in ED subjects.

Abbreviation: ED, emergency department; VAS, Visual Analog Scale.

and two subjects received $6 \mathrm{mg}$ MEQ. Other subjects received acetaminophen or nonsteroidal anti-inflammatory drugs. Drugs were administered via intramuscular or intravenous route in $42 \%$ of cases with no difference between opioids (40\%) and nonopioids (44\%).

ED treatment decreased pain scores and reduced nociception according to QST measures (Table 4). PPR was lower at $50 \mathrm{~N} / \mathrm{cm}^{2}(p=0.022)$ and $75 \mathrm{~N} / \mathrm{cm}^{2}(p=0.011)$, and PPT was higher $(p=0.0054)$. However, the absolute magnitude of change for each of these measures was small (Cohen's $\mathrm{d}_{\mathrm{z}}$ 0.10-0.19, Table 4). We did not detect any correlation between changes in ED pain scores and baseline QST measures $(r=0.05-0.13)$ or between changes in ED pain score and changes in PPT ( $\mathrm{r}=-0.11)$, PPR to $50 \mathrm{~N} / \mathrm{cm}^{2}(\mathrm{r}=0.12)$ or PPR to $75 \mathrm{~N} / \mathrm{cm}^{2}(\mathrm{r}=0.04)$.

We explored effect sizes for ED pain score or QST measures measured before and after treatment varied within clinical subgroups of subjects (Table 5). Effect sizes for QST measures did not differ consistently between sexes, pain characters, pain locations, analgesic type, route, or scores on PHQ-2 and GAD-2. However, effects on QST measures were medium in subjects with a history of or current treatment for anxiety and depression (Cohen's $d_{z}$ 0.26-0.43). Effect sizes were larger for ED pain score and medium to large (Cohen's d $\mathrm{z}_{\mathrm{z}}$.40-0.88) for QST measures in subjects with initial hyperalgesia (PPR to $50 \mathrm{~N} / \mathrm{cm}^{2}$ $\geq 9 \mathrm{~cm}$ ) (Table 5).

\section{Discussion}

We characterized a brief QST battery to measure pain perception in ED patients. All of the QST tests were reliable, confirming prior work. ${ }^{27}$ In healthy volunteers, oxycodone administration changed both PPT and PPR in a consistent manner, demonstrating sensitivity to interventions. The PPT and PPR tests require an inexpensive and portable algometer and $<1$ minute to complete, which are advantages for studies in the ED setting compared with more extensive QST batteries. ${ }^{28}$ Future studies may determine if other components of more extensive QST batteries, such as mechanical pain sensitivity, dynamic allodynia, and heat thresholds, provide 
Table 4 Analgesic treatment effect at 30 minutes on painful complaint and on QST in ED subjects $(n=173)$

\begin{tabular}{|c|c|c|c|c|}
\hline QST Measure & Baseline & $\begin{array}{l}\text { Post- } \\
\text { treatment }\end{array}$ & $\begin{array}{l}\text { Mean difference } \\
(95 \% \mathrm{Cl})\end{array}$ & Cohen's d \\
\hline Median (IQR): Verbal Pain Scale for ED complaint & $8(7,10)$ & $6(4,8)$ & $-2.2(-2.6,-1.9)$ & 0.97 \\
\hline Mean (SD): PPT, N & $47(18)$ & $49(19)$ & $2.5(0.5,4.5)$ & 0.19 \\
\hline Mean (SD): PPR $50 \mathrm{~N} / \mathrm{cm}^{2}$ VAS, $\mathrm{mm}$ & $52(28)$ & $49(29)$ & $-2.6(-6.4,1.2)$ & 0.10 \\
\hline Mean (SD): PPR $75 \mathrm{~N} / \mathrm{cm}^{2}$ VAS, $\mathrm{mm}$ & $73(29)$ & $69(31)$ & $-3.7(-7.1,-0.3)$ & 0.16 \\
\hline
\end{tabular}

Abbreviations: $\mathrm{Cl}$, confidence intervals; ED, emergency department; IQR, interquartile ranges; PPR, pressure pain response; PPT, pressure pain threshold; QST, quantitative sensory testing; SD, standard deviation; VAS, Visual Analog Scale.

Table 5 Analgesic treatment effect on painful complaint and on QST in subgroups of ED subjects ( $n=173$ )

\begin{tabular}{|c|c|c|c|c|c|c|c|c|c|}
\hline $\begin{array}{l}\text { Subject } \\
\text { characteristics }\end{array}$ & $\mathbf{n}$ & $\begin{array}{l}\text { Mean difference } \\
(95 \% \mathrm{Cl}), \mathrm{ED} \\
\text { Pain Scale* }\end{array}$ & $\begin{array}{l}\text { Cohen's } \\
d_{z}\end{array}$ & $\begin{array}{l}\text { Mean } \\
\text { difference } \\
(95 \% \mathrm{CI}) \\
\text { PPT, N }\end{array}$ & $\begin{array}{l}\text { Cohen's } \\
d_{z}\end{array}$ & $\begin{array}{l}\text { Mean difference } \\
(95 \% \mathrm{CI}) \\
\text { PPR } 50 \mathrm{~N} \\
\text { VAS, } \mathrm{mm}\end{array}$ & $\begin{array}{l}\text { Cohen's } \\
d_{z}\end{array}$ & $\begin{array}{l}\text { Mean difference } \\
(95 \% \mathrm{CI}) \\
\text { PPR } 75 \mathrm{~N} \\
\text { VAS, } \mathrm{mm}\end{array}$ & $\begin{array}{l}\text { Cohen's } \\
d_{z}\end{array}$ \\
\hline Male & 64 & $-2.2(-2.8,-1.8)$ & 0.94 & $2.2(-0.8,5.3)$ & 0.18 & $-4.1(-10,2.0)$ & 0.17 & $-2.5(-8.0,3.1)$ & 0.11 \\
\hline Female & 109 & $-2.3(-2.7,-1.7)$ & 1.00 & $2.7(0.0,5.3)$ & 0.19 & $-1.7(-6.6,3.3)$ & 0.06 & $-4.4(-8.8,0.0)$ & 0.19 \\
\hline \multicolumn{10}{|l|}{ Pain character } \\
\hline Musculoskeletal & 82 & $-1.8(-2.4,-1.3)$ & 0.78 & $3.5(0.8,6.2)$ & 0.28 & $-3.7(-8.9,1.4)$ & 0.16 & $-3.3(-9.0,2.4)$ & 0.13 \\
\hline Visceral & 70 & $-2.5(-3.0,-1.9)$ & 1.20 & I.I $(-2.6,4.8)$ & 0.07 & $0.1(-6.5,6.7)$ & 0.00 & $-3.8(-8.7,1.1)$ & 0.19 \\
\hline Soft tissue & 14 & $-3.0(-4.4,-1.6)$ & 1.24 & $3.2(-2.3,8.7)$ & 0.34 & $-2.4(-2.0,1.5)$ & 0.08 & $1.0(-8.5,10.4)$ & 0.06 \\
\hline \multicolumn{10}{|l|}{ Pain location } \\
\hline Extremities & 64 & $-2.1(-2.7,-1.4)$ & 0.84 & $2.7(-0.6,6.0)$ & 0.20 & $-5.2(-11,0.6)$ & 0.22 & $-5.0(-12,1.6)$ & 0.19 \\
\hline Abdominal & 65 & $-2.2(-2.7,-1.6)$ & 0.98 & I.7 $(-2.2,5.7)$ & 0.11 & $-1.3(-8.6,6.0)$ & 0.04 & $-5.9(-11,0.9)$ & 0.29 \\
\hline Back & 60 & $-2.2(-2.8,-1.6)$ & 0.98 & $2.0(-1.7,5.7)$ & 0.14 & $0.5(-6 . I, 7.0)$ & 0.02 & $-2.0(-6.5,2.5)$ & 0.12 \\
\hline Head & 21 & $-2.4(-3.3,-1.4)$ & 1.13 & $-0.5(-6.6,5.7)$ & 0.04 & $-7.8(-16,0.4)$ & 0.43 & $1.6(-8.8,12)$ & 0.07 \\
\hline Neck & 20 & $-2.2(-3.3,-1.0)$ & 0.84 & $2.4(-2.0,6.8)$ & 0.26 & $-7.3(-16,1.8)$ & 0.37 & $-2.5(-17,12)$ & 0.08 \\
\hline Chest & 11 & $-2.6(-4.6,-0.7)$ & 0.91 & $3.0(-5.5,11)$ & 0.23 & $-4.6(-18,9.1)$ & 0.23 & $3.6(-11,18)$ & 0.17 \\
\hline \multicolumn{10}{|l|}{ Treatment } \\
\hline Opioid & 101 & $-2.5(-2.9,-2.0)$ & 1.01 & $3.6(1.2,6.0)$ & 0.30 & $-I . I(-6.6,4.3)$ & 0.04 & $-4.6(-8.9,-0.2)$ & 0.21 \\
\hline Nonopioid & 72 & $-1.9(-2.4,-1.4)$ & 0.91 & I.0 $(-2.4,4.4)$ & 0.07 & $-4.6(-9.9,0.7)$ & 0.20 & $-2.5(-8.1,3.2)$ & 0.10 \\
\hline \multicolumn{10}{|l|}{ Opioid dose } \\
\hline $2 \mathrm{MEQ}$ & 64 & $-2.3(-2.9,-1.7)$ & 0.91 & $3.5(0.5,6.5)$ & 0.29 & $-0.1(-7.2,7.0)$ & 0.00 & $-2.1(-8.1,4.0)$ & 0.09 \\
\hline 4 MEQ & 34 & $-2.9(-3.7,-2.1)$ & 1.22 & $4.2(-0.2,8.7)$ & 0.33 & $-4.1(-14,5.4)$ & 0.15 & $-8.8(-15,-2.8)$ & 0.51 \\
\hline \multicolumn{10}{|l|}{ Drug route } \\
\hline Oral & 101 & $-1.9(-2.4,-1.5)$ & 0.82 & $2.6(-0.2,5.3)$ & 0.19 & $-0.1(-0.6,0.4)$ & 0.04 & $-0.2(-0.7,0.3)$ & 0.08 \\
\hline IV or IM & 72 & $-2.7(-3.2,-2.2)$ & 1.20 & $2.4(-0.5,5.4)$ & 0.19 & $-0.5(-1 . I, 0.2)$ & 0.18 & $-0.6(-1.1,-0.1)$ & 0.31 \\
\hline \multicolumn{10}{|c|}{ Anxiety/depression } \\
\hline Prior history & 90 & $-2.4(-2.9,-1.8)$ & 0.99 & $5.3(2.4,8.2)$ & 0.40 & $-6.3(-12,-0.8)$ & 0.26 & $-7.9(-13,-3.1)$ & 0.37 \\
\hline No history & 80 & $-2.1(-2.6,-1.7)$ & 0.94 & $0.1(-2.7,2.8)$ & 0.00 & $0.7(-4.9,6.4)$ & 0.03 & $-0.1(-5.1,4.9)$ & 0.00 \\
\hline \multicolumn{10}{|c|}{ Anxiety/depression } \\
\hline Current & 44 & $-2.7(-3.5,-2.0)$ & 1.13 & $5.1(0.6,9.6)$ & 0.34 & $-9.3(-16,-2.7)$ & 0.43 & $-8.8(-15,-2.6)$ & 0.43 \\
\hline None & 126 & $-2.1(-2.5,-1.7)$ & 0.91 & $1.6(-0.6,3.8)$ & 0.13 & $-0.2(-4.9,4.5)$ & 0.01 & $-2.0(-6.2,2.2)$ & 0.08 \\
\hline PHQ-2 >3 & 60 & $-2.1(-2.6,-1.5)$ & 0.94 & $3.7(0.5,7.0)$ & 0.30 & $-0.2(-0.8,0.4)$ & 0.10 & $-0.4(-0.8,0.1)$ & 0.19 \\
\hline PHQ-2 $\leq 3$ & 113 & $-2.3(-2.8,-1.9)$ & 0.98 & $1.9(-0.7,4.4)$ & 0.10 & $-0.3(-0.8,0.2)$ & 0.10 & $-0.4(-0.9,0.1)$ & 0.15 \\
\hline GAD-2 >3 & 69 & $-2.1(-2.8,-1.8)$ & 1.02 & $4.3(1.1,7.6)$ & 0.32 & $-0.1(-0.7,0.5)$ & 0.05 & $-0.3(-0.8,0.1)$ & 0.15 \\
\hline GAD-2 $\leq 3$ & 104 & $-2.3(-2.7,-1.6)$ & 0.93 & $1.3(-1.2,3.8)$ & 0.10 & $-0.3(-0.9,0.2)$ & 0.13 & $-0.4(-0.9,0.2)$ & 0.17 \\
\hline \multicolumn{10}{|l|}{ Hyperalgesia } \\
\hline PPR $50 \mathrm{~N} / \mathrm{cm}^{2}>9$ & 23 & $-3.3(-4.5,-2.1)$ & 1.21 & $3.6(-0.0,7.2)$ & 0.43 & $-2.0(-3.0,1.0)$ & 0.88 & $-0.4(-0.9,0.0)$ & 0.40 \\
\hline PPR $50 \mathrm{~N} / \mathrm{cm}^{2}<9$ & 150 & $-2.1(-2.4,-1.7)$ & 0.94 & $2.3(0.1,4.6)$ & 0.17 & $0.0(-0.4,0.4)$ & 0.01 & $-0.4(-0.7,0.0)$ & 0.15 \\
\hline
\end{tabular}

Notes: *Second value missing for three subjects.

Abbreviations: $\mathrm{Cl}$, confidence intervals; ED, emergency department; GAD-2, Generalized Anxiety Disorder scale-2; IM, intramuscular; IV, intravenous; MEQ, morphine equivalent dose; n, number of subjects; PHQ-2, Patient Health Questionnaire-2; PPR, pressure pain response; PPT, pressure pain threshold; QST, quantitative sensory testing; VAS, Visual Analog Scale.

additional information in ED patients. Over $88 \%$ of ED patients completed repeat testing while being treated for painful conditions, demonstrating the feasibility of QST in the chaotic ED environment. PPT and PPR also change with
ED analgesic drug treatment in parallel with the reported pain from the ED complaint.

We are aware of no other studies that used QST to study treatment response in the ED. One study in surgical patients 
found QST measures, primarily lower electrical and heat pain thresholds, were associated with higher postoperative analgesic requirements. ${ }^{29}$ Recent studies suggest that QST measures can identify interindividual differences in particular mechanisms of pain perception, such as descending-inhibitory activity, which can explain responses to specific types of drugs. ${ }^{30,31}$ Our study demonstrates the feasibility of similarly measuring interindividual variation in ED patients. These techniques could guide personalized selection of specific drugs or therapies for acute pain treatment and could identify subgroups of patients with differential response (Table 5).

CPT and PST were less suitable for the ED setting. CPT tests required ice water and created some perceived change in the skin of the subject's hand for up to 10 minutes that might affect repeated tests. ${ }^{32}$ We used the simplest possible apparatus for CPT testing, which did not include any effort to circulate water around the hand. Therefore, a pocket of warmer water may have developed around each subject's hand, increasing variability in response. PST required several minutes to complete and was less reliable between raters. Therefore, we did not attempt to collect these measures in ED subjects. Automated devices to test temperature pain thresholds or sensory thresholds might be more reliable but are not yet available for application in large-scale research trials or routine ED practice. ${ }^{33}$

Our data confirm that verbal numerical pain scores are highly correlated with the more granular VAS. ${ }^{16}$ Verbal numerical scales will be more practical for telephone or remote monitoring of pain after emergency care. ${ }^{34-36}$ Prior research indicates that a change of $1.3-1.6 \mathrm{~cm}$ in VAS is clinically important to ED patient experience. ${ }^{17-19}$ The $95 \%$ CIs for intrasubject and inter-rater agreement in our healthy volunteers (Table 1) confirm that changes in that magnitude are unlikely to be testing error. Most of our ED subjects reported changes in pain score greater than this threshold (Table 4). Enteral versus parenteral route of drug administration did not alter the analgesic effects (Table 5), consistent with a prior ED study that parenteral drugs have rapid onset but indistinguishable effects after 30-40 minutes. ${ }^{37}$

Our data suggest that factors in addition to reduced perception of painful stimuli contribute to the clinical efficacy of ED pain treatment. Specifically, the change in PPT and PPR with treatment explained little variation in ED pain score $(r=0.04-0.12)$. ED subjects also reported reductions in the severity of their painful complaint after only 30 minutes (Table 4) although analgesic effects of oxycodone were not detectable in fasting healthy volunteers until 60 minutes (Figure 3). One possible explanation of these results is that reducing patient anxiety, setting expectations for relief, and providing non-pharmacological support are amplifiers of the effect of ED analgesics and may take effect much faster than drugs. These treatments may increase the ability to tolerate and endure pain of a given intensity (i.e., reduce discomfort), despite small changes in perceived pain. While distinguishing nociception from discomfort was not the primary focus of this study, future research should use more than the single numerical rating scale to assess the painful ED complaint. ${ }^{20}$ In addition, clinicians should exploit multiple different mechanisms to reduce or optimize total drug required for effective acute pain treatment.

Our results support a contribution of ED patient psychological traits to the pain treatment response. A history of treatment for anxiety or depression was associated with stronger effects of ED analgesic administration on QST measures. Anxiety and affective disorders are comorbid with chronic pain, ${ }^{14,15}$ modify severity of postsurgical pain, ${ }^{38}$ and impair central pain inhibition in chronic visceral pain. ${ }^{39}$ The fact that ED patients with a history of anxiety or depression were more responsive to ED treatment appears paradoxical. However, it is possible that these traits increase patient response to stimuli, as in the prior studies, creating a larger opportunity for acute ED treatment of nociception to reduce pain perception. Alternatively, many drugs used in the ED also reduce anxiety or provide euphoria that could reduce state anxiety or negative affect. The GAD-2 and PHQ-2 were not associated with analgesic effect, perhaps because these instruments are too brief to identify patient anxiety and mood (Table 5). Alternatively, state anxiety or mood may modify pain response less than trait anxiety or mood. More detailed phenotyping of the ED subjects using instruments that can separate state, trait, affective and cognitive modifiers of pain response will be required to understand the differential response to treatment in these subgroups. ${ }^{40}$

Hypersensitivity (lower PPT) and hyperresponsiveness (stronger PPR) outside the range observed in healthy volunteers occurred in at least $16 \%$ of ED patients (Figure 3 ). Similar patterns occur in patients with chronic pain. ${ }^{41-43}$ Hyperalgesia was unlikely to be an artifact of QST administration because we used the same testers and same equipment in both ED and healthy volunteer cohorts. The prevalence of hyperalgesia has not previously been described in an acute ED population. Our definition of this subgroup is arbitrarily based on the distributions of PPR in our cohorts, and estimated prevalence would differ with different operational definitions. Nevertheless, demonstration that this subgroup exists and can be detected during ED care allows future research to characterize its features. 
Multiple factors may cause hyperalgesia in ED subjects. The higher incidence of anxiety or depression history in the hyperalgesic subgroup suggests contribution of psychological traits of the subjects and resembles the co-incidence of these problems in other cohorts with chronic pain..$^{14,15,44}$ Alternatively, the ED painful complaint may sensitize the subject to other stimuli as can happen with neuropathic pain. ${ }^{42}$ Finally, some ED complaints may be acute exacerbations of neuropathic pain and some ED patients may have prior opioid treatment, both of which can cause hyperalgesia. ${ }^{45}$ Response to QST measures in these subjects at other times outside of the ED encounter could inform to what extent hyperalgesia is a trait or situational phenomenon, and responses in multiple anatomical regions could inform about the mechanisms. In addition, future studies should more precisely define the duration or chronicity of the ED complaint.

An important limitation of the ED study was the observational design in which subjects were not assigned to receive drug versus placebo. Therefore, the change in measures before and after treatment could also be attributed to placebo effect, secular trend, or regression to the mean. The larger change in QST measures and ED pain scores in the subset of hyperalgesic subjects (Table 5) may represent regression towards the population mean. This subset of subjects was defined post hoc by extreme scores on the baseline QST measures, and any statistical difference in the change of measures is suspect. Nevertheless, it is tempting to speculate that ED treatment more effectively reduces the sensitization contributions to pain perception in these subjects. We would need to confirm any differential effect of ED treatment on central sensitization in a prospective study that could adjust for initial pain thresholds.

These data illustrate that interindividual differences in perception of or tolerance to fixed stimuli contribute to acute pain perception and analgesic response in the ED. Making QST measurements concurrently with assessment of ED pain scores provides the ability to separate the contribution of perception to total relief of discomfort. Opportunities to use this information might include selection of drugs with fewer side effects than opioids to provide anxiolysis in patients where anxiety is present. ${ }^{46}$ Anxiolysis and polypharmacy are already common practice in ED procedural sedation. ${ }^{47}$ Providers could use behavioral or cognitive-behavioral strategies to encourage cognitive reframing of the pain and augment the effects of acute analgesics. ${ }^{48,49}$ These data might allow rational multimodal ED pain regimens personalized for each patient. This methodological refinement also allows more mechanistic examination of subjective drug effects and identification of treatment-responsive subgroups.
In conclusion, a QST battery reveals individual variation in ED patient response to pain with $\sim 16 \%$ of ED patients exhibiting pressure hyperalgesia relative to healthy volunteers. Treatment in the ED produces a large reduction in the pain experienced by subjects with only small effects on objective nociception, suggesting multiple contributions to the reported ED pain. Subjects with a history of treatment for anxiety or depression or initial hyperalgesia are particularly responsive to ED drug treatment. These data demonstrate opportunities to select personalized and multimodal treatment for ED painful complaints.

\section{Acknowledgments}

This work was supported by the Department of Emergency Medicine, University of Pittsburgh.

\section{Disclosure}

The authors report no conflicts of interest in this work.

\section{References}

1. Mazer-Amirshahi M, Mullins PM, Rasooly I, van den Anker J, Pines JM. Rising opioid prescribing in adult U.S. emergency department visits: 2001-2010. Acad Emerg Med. 2014;21(3):236-243.

2. Hoppe JA, Nelson LS, Perrone J, Weiner SG, Prescribing Opioids Safely in the Emergency Department (POSED) Study Investigators. Opioid prescribing in a cross section of US emergency departments. Ann Emerg Med. 2015;66(3):253-259.

3. Kea B, Fu R, Lowe RA, Sun BC. Interpreting the National Hospital Ambulatory Medical Care Survey: United States emergency department opioid prescribing, 2006-2010. Acad Emerg Med. 2016;23(2):159-165.

4. Butler MM, Ancona RM, Beauchamp GA, et al. Emergency department prescription opioids as an initial exposure preceding addiction. Ann Emerg Med. 2016;68(2):202-208.

5. Hoppe JA, Houghland J, Yaron M, Heard K. Prescription history of emergency department patients prescribed opioids. West J Emerg Med. 2013;14(3):247-252.

6. Burton JH, Hoppe JA, Echternach JM, Rodgers JM, Donato M. Quality improvement initiative to decrease variability of emergency physician opioid analgesic prescribing. West J Emerg Med. 2016;17(3):258-263.

7. Weiner SG, Raja AS, Bittner JC, et al. Opioid-related policies in New England emergency departments. Acad Emerg Med. 2016;23(9):1086-1090.

8. Heins A, Grammas M, Heins JK, Costello MW, Huang K, Mishra S. Determinants of variation in analgesic and opioid prescribing practice in an emergency department. J Opioid Manag. 2006;2(6):335-340.

9. Miner J, Biros MH, Trainor A, Hubbard D, Beltram M. Patient and physician perceptions as risk factors for oligoanalgesia: a prospective observational study of the relief of pain in the emergency department. Acad Emerg Med. 2006;13(2):140-146.

10. Pollack CV Jr, Viscusi ER. Improving acute pain management in emergency medicine. Hosp Pract. 2015;43(1):36-45.

11. Singer AJ, Garra G, Chohan JK, Dalmedo C, Thode HC Jr. Triage pain scores and the desire for and use of analgesics. Ann Emerg Med. 2008;52(6):689-695.

12. Edwards RR, Dworkin RH, Turk DC, et al. Patient phenotyping in clinical trials of chronic pain treatments: IMMPACT recommendations. Pain. 2016;157(9):1851-1871.

13. Kapoor S, White J, Thorn BE, Block P. Patients presenting to the emergency department with acute pain: the significant role of pain catastrophizing and state anxiety. Pain Med. 2016;17(6):1069-1078. 
14. McWilliams LA, Cox BJ, Enns MW. Mood and anxiety disorders associated with chronic pain: an examination in a nationally representative sample. Pain. 2003;106(1-2):127-133.

15. McWilliams LA, Goodwin RD, Cox BJ. Depression and anxiety associated with three pain conditions: results from a nationally representative sample. Pain. 2004;111(1-2):77-83.

16. Bijur PE, Latimer CT, Gallagher EJ. Validation of a verbally administered numerical rating scale of acute pain for use in the emergency department. Acad Emerg Med. 2003;10(4):390-392.

17. Gallagher EJ, Bijur PE, Latimer C, Silver W. Reliability and validity of a visual analog scale for acute abdominal pain in the ED. Am JEmerg Med. 2002;20(4):287-290.

18. Gallagher EJ, Liebman M, Bijur PE. Prospective validation of clinically important changes in pain severity measured on a visual analog scale. Ann Emerg Med. 2001;38(6):633-638.

19. Todd KH, Funk KG, Funk JP, Bonacci R. Clinical significance of reported changes in pain severity. Ann Emerg Med. 1996;27(4):485-489.

20. Krebs EE, Carey TS, Weinberger M. Accuracy of the pain numeric rating scale as a screening test in primary care. $J$ Gen Intern Med. 2007;22(10):1453-1458.

21. Kroenke K, Spitzer RL, Williams JB. The patient health questionnaire-2: validity of a two-item depression screener. Med Care. 2003;41(11):1284-1292.

22. Plummer F, Manea L, Trepel D, McMillan D. Screening for anxiety disorders with the GAD-7 and GAD-2: a systematic review and diagnostic metaanalysis. Gen Hosp Psychiatry. 2016;39:24-31.

23. Patanwala AE, Duby J, Waters D, Erstad BL. Opioid conversions in acute care. Ann Pharmacother. 2007;41(2):255-266.

24. Pereira J, Lawlor P, Vigano A, Dorgan M, Bruera E. Equianalgesic dose ratios for opioids. a critical review and proposals for long-term dosing. J Pain Symptom Manage. 2001;22(2):672-687.

25. Bland JM, Altman DG. Statistical methods for assessing agreement between two methods of clinical measurement. Lancet. 1986;1(8476):307-310.

26. Lakens D. Calculating and reporting effect sizes to facilitate cumulative science: a practical primer for t-tests and ANOVAS. Front Psychol. 2013;4:863.

27. Geber C, Klein T, Azad S, et al. Test-retest and interobserver reliability of quantitative sensory testing according to the protocol of the German Research Network on Neuropathic Pain (DFNS): a multi-centre study. Pain. 2011;152(3):548-556.

28. Rolke R, Magerl W, Campbell KA, et al. Quantitative sensory testing: a comprehensive protocol for clinical trials. Eur J Pain. 2006;10(1): 77-88.

29. Grosen K, Fischer IW, Olesen AE, Drewes AM. Can quantitative sensory testing predict responses to analgesic treatment? Eur J Pain. 2013;17(9):1267-1280.

30. Cruz-Almeida Y, Fillingim RB. Can quantitative sensory testing move us closer to mechanism-based pain management? Pain Med. 2014;15(1):61-72.

31. Edwards RR, Dolman AJ, Martel MO, et al. Variability in conditioned pain modulation predicts response to NSAID treatment in patients with knee osteoarthritis. BMC Musculoskelet Disord. 2016;17:284

32. Gröne E, Crispin A, Fleckenstein J, Irnich D, Treede RD, Lang PM. Test order of quantitative sensory testing facilitates mechanical hyperalgesia in healthy volunteers. J Pain. 2012;13(1):73-80.

33. Harte SE, Mitra M, Ichesco EA, et al. Development and validation of a pressure-type automated quantitative sensory testing system for pointof-care pain assessment. Med Biol Eng Comput. 2013;51(6):633-644.
34. Chang AK, Bijur PE, Holden L, Gallagher EJ. Comparative analgesic efficacy of oxycodone/acetaminophen versus hydrocodone/acetaminophen for short-term pain management in adults following ED discharge. Acad Emerg Med. 2015;22(11):1254-1260.

35. Chang AK, Bijur PE, Lupow JB, Gallagher EJ. Comparative analgesic efficacy of oxycodone/acetaminophen versus codeine/acetaminophen for short-term pain management following ED discharge. Pain Med. 2015;16(12):2397-2404.

36. Suffoletto B, Wagner AK, Arenth PM, et al. Mobile phone text messaging to assess symptoms after mild traumatic brain injury and provide self-care support: a pilot study. J Head Trauma Rehabil. 2013;28(4):302-312.

37. Miner JR, Moore J, Gray RO, Skinner L, Biros MH. Oral versus intravenous opioid dosing for the initial treatment of acute musculoskeletal pain in the emergency department. Acad Emerg Med. 2008;15(12):1234-1240.

38. Thomazeau J, Rouquette A, Martinez V, et al. Acute pain factors predictive of post-operative pain and opioid requirement in multimodal analgesia following knee replacement. Eur J Pain. 2016;20(5):822-832.

39. Piché M, Bouin M, Arsenault M, Poitras P, Rainville P. Decreased pain inhibition in irritable bowel syndrome depends on altered descending modulation and higher-order brain processes. Neuroscience. 2011;195:166-175.

40. Lautenbacher S, Huber C, Kunz M, et al. Hypervigilance as predictor of postoperative acute pain: its predictive potency compared with experimental pain sensitivity, cortisol reactivity, and affective state. Clin J Pain. 2009;25(2):92-100.

41. Defrin R, Schreiber S, Ginzburg K. Paradoxical pain perception in posttraumatic stress disorder: the unique role of anxiety and dissociation. J Pain 2015;16(10):961-970.

42. Konopka KH, Harbers M, Houghton A, et al. Bilateral sensory abnormalities in patients with unilateral neuropathic pain; a quantitative sensory testing (QST) study. PLoS One. 2012;7(5):e37524.

43. Tesarz J, Gerhardt A, Leisner S, Janke S, Treede RD, Eich W. Distinct quantitative sensory testing profiles in nonspecific chronic back pain subjects with and without psychological trauma. Pain. 2015;156(4):577-586.

44. Wasan AD, Michna E, Edwards RR, et al. Psychiatric comorbidity is associated prospectively with diminished opioid analgesia and increased opioid misuse in patients with chronic low back pain. Anesthesiology. 2015;123(4):861-872.

45. Friedman B, Bijur P, Greenwald P, Lipton R, Gallagher EJ. Clinical significance of brush allodynia in emergency patients with migraine. Headache. 2009;49(1):31-35.

46. Behrbalk E, Halpern P, Boszczyk BM, et al. Anxiolytic medication as an adjunct to morphine analgesia for acute low back pain management in the emergency department: a prospective randomized trial. Spine. 2014;39(1):17-22.

47. Bellolio MF, Gilani WI, Barrionuevo P, et al. Incidence of adverse events in adults undergoing procedural sedation in the emergency department: a systematic review and meta-analysis. Acad Emerg Med. 2016;23(2):119-134.

48. Morley S. Efficacy and effectiveness of cognitive behaviour therapy for chronic pain: Progress and some challenges. Pain. 2011;152(3 Suppl): S99-S106.

49. Morley S, Eccleston C, Williams A. Systematic review and metaanalysis of randomized controlled trials of cognitive behaviour therapy and behavior therapy for chronic pain in adults, excluding headache. Pain. 1999;80(1-2):1-13. 
The Journal of Pain Research is an international, peer reviewed, open access, online journal that welcomes laboratory and clinical findings in the fields of pain research and the prevention and management of pain. Original research, reviews, symposium reports, hypothesis formation and commentaries are all considered for publication
The manuscript management system is completely online and includes a very quick and fair peer-review system, which is all easy to use. Visit http://www.dovepress.com/testimonials.php to read real quotes from published authors.

Submit your manuscript here: https://www.dovepress.com/journal-of-pain-research-journal 